See discussions, stats, and author profiles for this publication at: https://www.researchgate.net/publication/1781396

\title{
Localized Structures Embedded in the Eigenfunctions of Chaotic Hamiltonian Systems
}

Article in Physical review. E, Statistical physics, plasmas, fluids, and related interdisciplinary topics · April 1998

DOI: 10.1103/PhysRevE.58.R5225 · Source: arXiv

CITATIONS

READS

11

14

2 authors, including:

D. A. Wisniacki

University of Buenos Aires

80 PUBLICATIONS 814 CITATIONS

SEE PROFILE 


\title{
Localized Structures Embedded in the Eigenfunctions of Chaotic Hamiltonian Systems
}

\author{
E. Vergini ${ }^{a}$ and D.A. Wisniacki ${ }^{b}$ \\ ${ }^{1}$ Departamento de Física, Comisión Nacional de Energía Atómica. Av. del Libertador 8250, 1429 Buenos Aires, Argentina. \\ ${ }^{2}$ Departamento de Física "J.J. Giambiagi", FCEN, UBA, Pabellón 1, Ciudad Universitaria, 1428 Buenos Aires, Argentina
}

(February 5, 2008)

We study quantum localization phenomena in chaotic systems with a parameter. The parametric motion of energy levels proceeds without crossing any other and the defined avoided crossings quantify the interaction between states. We propose the elimination of avoided crossings as the natural mechanism to uncover localized structures. We describe an efficient method for the elimination of avoided crossings in chaotic billiards and apply it to the stadium billiard. We find many scars of short periodic orbits revealing the skeleton on which quantum mechanics is built. Moreover, we have observed strong interaction between similar localized structures.

PACS numbers: 05.45.+b, 03.65.Sq, 03.20.+i

The connection between individual eigenstates and classical invariant sets in chaotic systems is far from being fully understood at present. A first step was given by Berry [1] and Voros [2] twenty years ago. They conjectured that the eigenstates are associated with the whole energy surface explored ergodically by the orbits and then, the eigenstates would be locally like random superpositions of plane waves. That picture is supported by Shnirelman's theorem [3] and the pioneering numerical work by McDonald and Kaufman [-1] on the Bunimovich stadium billiard - an ergodic system [5]. However, the paper by Heller [6] modified that point of view. He emphasized that a large number of high excited eigenfunctions for the stadium billiard has an enhancement along the shorter periodic trajectories (scars). In 1988, Bogomolny [7] developed the semiclassical theory of scars and explained the extra density near unstable periodic trajectories. He represented the probability density as a sum over a finite number of periodic orbits (see also [8]).

Another unexpected localization phenomenon was observed in the stadium billiard for the first time; the famous bouncing ball (b.b) states [9]. They correspond to the family of neutral periodic orbits living in the stadium. Localization is stronger than in the case of scars and survives the semiclassical limit [10]. The semiclassical theory of b.b was developed recently by Tanner [11].

It is easy to observe b.b states in the stadium billiard, but it is practically impossible to find complete series of b.b states. On the other hand, it is very difficult to find a family of scars. That is, given a scar with wave number $k$ which is associated to a particular periodic orbit of lenght $\mathcal{L}$, we expect to see a family of scars of the same orbit at $k+2 \pi n / \mathcal{L}$ [6], with $n$ an integer. But in general, a sequence of similar scars is not observed. Such a phenomenon appears only for some short periodic orbits in some regions of the spectrum but it is impossible to define a systematic of scars 12.

We believe that in order to study the mechanisms giving rise to localization it is necessary first to clean the spectrum. The individual properties of two well defined states depending on a parameter are mixed when they collide in an avoided crossing [13]. Then, we expect that the elimination of avoided crossings (a.c) in systems governed by a parameter dependent Hamiltonian provides a mechanism for localization. But in a chaotic system it is impossible to remove all a.c because an individual eigenstate interacts strongly with other states and the interaction is of long range. However, we will extract only the a.c with highest curvatures. Recently, it was stressed 14 that the distribution of highest curvatures has a universal character and the tail of low curvatures is a particular property of the system possibly associated with scarring phenomena. Precisely, we want to remove the universal property of chaotic eigenfunctions related to the Shnirelman's theorem, and to retain fluctuations revealed like phase space localization. On the same footing, Takami 15] has suggested that a.c are contributed by long periodic orbits. In the light of this, by removing a.c, scars of short periodic orbits would arise.

We have developed a simple method to remove a.c in chaotic billiards governed by a shape parameter. Let's $\phi_{\mu}(\mathbf{r})$ and $\phi_{\nu}(\mathbf{r})$ be normalized eigenfunctions of a planar billiard with Dirichlet conditions on the boundary $\mathcal{C}$. In ref. [16] the following quasiorthogonality relation was shown

$$
\oint_{\mathcal{C}} \frac{\partial \phi_{\mu}}{\partial \mathbf{n}} \frac{\partial \phi_{\nu}}{\partial \mathbf{n}} \frac{r_{n} d s}{2 k_{\mu} k_{\nu}}=\delta_{\mu, \nu}+\frac{\left(k_{\mu}-k_{\nu}\right)}{\left(k_{\mu}+k_{\nu}\right)} \mathcal{O}(1)
$$

where $k_{\mu}$ and $k_{\nu}$ are the corresponding eigenwave numbers, $r_{n} \equiv \mathbf{r . n}$ with $\mathbf{n}$ the outward normal unit vector to $\mathcal{C}$, and $s$ is arc lenght round $\mathcal{C}$. Relation (11) defines an effective Hilbert space whose dimension is of order $k$, and where an eigenstate is represented by $\varphi(s) \equiv \partial \phi / \partial \mathbf{n}(\mathbf{r}(s))$; the eigenfunction in this space.

Now we deform the boundary by changing a shape parameter $\ell\left(\ell=\ell_{0}\right.$ for $\left.\mathcal{C}\right)$. Using equation (11) it is possible to show 17] that eigenenergies $\left(k^{2}\right)$ and eigenfunctions at different values of the parameter are connected through the following parameter dependent Hamiltonian written in the basis $\left\{\varphi_{\mu}\right\}$ of eigenfunctions at $\ell_{0}$,

$$
H_{\mu \nu}\left(\ell_{0}+\delta \ell\right) \simeq k_{\mu}^{2} \delta_{\mu, \nu}+\delta \ell H_{\mu \nu}^{\prime}
$$

with 


$$
H_{\mu \nu}^{\prime}=-\left(\oint_{\mathcal{C}} r_{n}^{\prime} \varphi_{\mu} \varphi_{\nu} d s\right) \times\left[1+\left(k_{\mu}-k_{\nu}\right) \mathcal{O}\left(k^{-1}\right)\right]
$$

where primes indicate derivation with respect to $\ell$. Relation (3) was obtained previously [18 for the case when $k_{\mu}=k_{\nu}$. By diagonalizing $H\left(\ell_{1}\right)$ we obtain the eigenenergies $\tilde{k}_{\mu}^{2}$ and the eigenfunctions $\tilde{\varphi}_{\mu}$ at $\ell_{1}$. Suppose that at $\ell_{1}$ the states $\mu$ and $\nu$ collide in an a.c. In ref. [19] an efficient way of determining a.c was described in terms of the coefficients $C_{\mu \nu}(\ell) \equiv<\varphi_{\mu} \mid \partial \varphi_{\nu} / \partial \ell>$ which define a driven evolution of the system. Using equation (2) and perturbation theory we obtain $C_{\mu \nu}(\ell)=H_{\mu \nu}^{\prime} /\left(k_{\mu}^{2}-k_{\nu}^{2}\right)$, where $\mu$ and $\nu$ are associated to eigenstates of $H(\ell)$ (the adiabatic basis). Around $\ell_{1}, C_{\mu \nu}$ behaves like the Lorentzian function $\left(\ell_{i n t} / 2\right) /\left[\ell_{i n t}^{2}+\left(\ell-\ell_{1}\right)^{2}\right]$, with $\ell_{\text {int }}=1 / 2 C_{\mu \nu}\left(\ell_{1}\right)$. The area in the energy spectrum, where the a.c is relevant, can be estimated by $A_{\mu \nu}=\Delta k^{2} \times \ell_{\text {int }}$. Then, if the area is lower than a prescribed value $v$

$$
A_{\mu \nu}=\left(k_{\mu}^{2}-k_{\nu}^{2}\right)^{2} /\left|H_{\mu \nu}^{\prime}\right|<v \equiv m \dot{\ell} \ln 2^{4} / \pi \hbar,
$$

we eliminate the a.c by transforming the diagonal matriz of $H\left(\ell_{1}\right)$ as follows: $\tilde{k}_{\mu}^{2}($ new $)=\tilde{k}_{\nu}^{2}($ new $)=\left(\tilde{k}_{\mu}^{2}+\tilde{k}_{\nu}^{2}\right) / 2$. All a.c which satisfy (4) are eliminated in the increasing order of their area. Criterion (4) has also a simple dynamic interpretation. If the billiard contains a particle of mass $m$ and the boundary is driven with a velocity $\dot{\ell}$, the a.c is eliminated when the Landau-Zener probability transition is greater than one half.

The new Hamiltonian is less chaotic than the previous one, and the adiabatic basis of the new Hamiltonian is more adequate to study nonadiabatic effects [20]. For nearly integrable billiards, this new adiabatic basis goes to the diabatic one of the original Hamiltonian. For chaotic billiards, the elimination of a.c between nearest neighboring levels gives rise to a.c between distant levels (see Fig. 2(a)).

We have studied the desymmetrized stadium billiard with radius $r$ and straight line of length $a$ 19. The boundary only depends on the shape parameter $\ell=a / r$ (the area is fixed to the value $1+\pi / 4$ ). Fig. 1 compares the approximated spectrum 21] obtained from eq. (2) with the exact one; the agreement is excellent. We find structures surviving parametric variation (straight lines interrupted by small a.c). The most evident being b.b states, e.g. states 6, 14 and 18 of Fig. B(a). But in general any localized eigenfunction has the same behavior. In Fig. 2(a) we show the spectrum of the transformed Hamiltonian by elimination of all a.c satisfying eq.(4), with $v=1.5$. At this stage all b.b structures embedded in the spectrum emerge clearly. b.b states are identified by the quantum numbers $\left(n_{x}, n_{y}\right)$ counting excitations in the horizontal and vertical direction respectively; $n_{y}$ labels b.b series. The states 14, 18 and 23 of Fig. 3(b) correspond to $\left(n_{x}, 15\right)$, with $n_{x}=1,2$ and 3 resp., and states 6,16 and 28 to $\left(n_{x}, 14\right)$, with $n_{x}=6,7$ and 8 . At this stage also many scars of short periodic orbits clearly appear. They are the states $1,2,4,7,8,9,12,17,20,21$, 22, 26, 28, 29 and 30 of Fig. 3(b); the associated periodic orbits are identified in Fig. 1. In Fig. 2(b) we have eliminated avoided crossings up to $v=5$ giving rise to new localized structures; states $3,5,10,11,13,24$ and 27 of Fig. 3(b). We have not identified the states 15 and 25 (probably contributed by the whispering gallery family of periodic orbits) and the state 19 .

The structures in Fig. 3(b) are obtained from the spectrum (Fig. 3(a)) by an orthogonal transformation which reduces the parametric interaction among states; that is , the non-diagonal elements of the deformation matrix $H^{\prime}$ are reduced considerably in the new basis. The new states are characterized by the mean energy $<k^{2}>$ and the dispersion $\sigma$ measured in units of the mean energy levels. If $\sigma$ is lower than one, the new state has high probability of appearing in the spectrum. For $\sigma=1$ the associated structure lives a time equal to the Heisenberg time. For scars of periodic trajectories we expect $\sigma$ to increase with $k$ according to the Shnirelman's theorem. For b.b states with fixed $n_{x}$, we expect $\sigma$ to go to zero when $n_{y}$ go to infinite.

The non-diagonal elements of the original Hamiltonian in the new basis give us the interaction between localized structures. We have observed strong interaction between similar structures. For example, b.b states $(1,15)$ and $(2,15)$ have low interaction with the other states (a.c are very small -see Fig. 1), except with the b.b state $(7,14)$ and with the states 10 and 24 of Fig. 3(b). Strong interaction was also observed between scars of short periodic orbits which are close in phase space. For example, states 4 and 22 have strong interaction with states 2 and 17 respectively, and states 10 and 24 with states 11 and 27 respectively. In some situations, this strong interaction probably decides the occurrence of a defined structure. For example, states 4 and 22 are built principally with orbit $(\mathbf{j})$, but the Bohr-Sommerfeld quantization rule for this orbit $(k=0.6999(n+1 / 4))$ predicts a scar at $k \sim$ 47.07 which is not observed. However, the second contribution, given by orbit $(\mathbf{d})(k=1.3013(n+1 / 2))$, does not predict such scar. In fact, in the range $35<k<55$ we have observed structures like 4 and 22 only for those values of $\mathrm{k}$ which satisfy approximatelly quantization for (j) and (d) simultaneously. A deep study of this beating phenomenon and the interaction between periodic orbits, which appear very strong and can introduce new insight into the semiclassical theory of chaotic systems, is presently under way [17].

We transform the eigenfunctions in order to reduce the parametric interaction between states. From a semiclassical point of view, the new structures are not contributed by long periodic orbits. The study of the new states in terms of short periodic orbits is amenable to the technique developed recently in ref. 12.

Finally, we mention that the large parametric corre- 
lation observed by Tomsovic 22 in the stadium billiard (random matrix theory predicts no correlations) is simply understood in terms of these localized structures. On the other hand, diffusion and dissipation in mesoscopic systems are affected by these localized structures and the theory developped by Wilkinson 23] based on random matrix theory needs to be revised.

We would like to thank M. Saraceno for useful suggestions. E.V. acknowledges the hospitality of U. Smilansky and The Weizmann Institute of Science where part of this work has been done.

[1] M. V. Berry, J. Phys. A 10, 2083 (1977).

[2] A. Voros, in Stochastic Behaviour in Classical and Quantum Hamiltonian Systems, eds. G. Casati and G. Ford, Lectures Notes in Physics 93, (Springer, Berlin, 1979) p. 326.

[3] A. I. Shnirelman, Usp. Mat. Nauk 29, 181 (1974).

[4] S. W. McDonald and A. N. Kaufman, Phys. Rev. Lett. 42, 1189 (1979).

[5] L. A. Bunimovich, Funct. Anal. Appl. 8, 254 (1974).

[6] E. J. Heller, Phys. Rev. Lett. 53, 1515 (1984).

[7] E. B. Bogomolny, Physica D 31, 169 (1988).

[8] M. V. Berry, Proc. R. Soc. London A 423, 219 (1989). O. Agam and S. Fishman, J. Phys. A 26, 2113 (1993).

[9] S. W. McDonald, Ph. D. Thesis UC berkeley LBL14837 (1983).

[10] P. W. O'Connor and E. J. Heller, Phys. Rev. Lett. 61, 2288 (1988).

[11] G. Tanner, J. Phys. A 30, 2863 (1997).

[12] F. Simonotti, E. Vergini and M. Saraceno, Phys. Rev. E 56, 3859 (1997).

[13] C. E. Zener, Proc. R. Soc. London Ser. A 137, 696 (1932).

[14] J. Zakrzewski and D. Delande, Phys. Rev. E 47, 1650 (1993).

[15] T. Takami, Phys. Rev. Lett. 68, 3371 (1992).

[16] E. Vergini and M. Saraceno, Phys. Rev. E 52, 2204 (1995).

[17] E. Vergini and D. A. Wisniacki (to be submitted).

[18] M. V. Berry and M. Wilkinson, Proc. R. Soc. London A 392, 15 (1984).

[19] M. J. Sánchez, E. Vergini, and D. A. Wisniacki, Phys. Rev. E 54, 4812 (1996).

[20] W. Lichten, Phys. Rev. 131, 229 (1963); F. T. Smith, ibid. 179, 111 (1969).

[21] We have multiplied the deformation matrix (eq. (3)) by the Lorentzian function $\left[1+\operatorname{Area}\left(k_{\mu}^{2}-k_{\nu}^{2}\right)\right]^{-1}$ to have into account the finite character of the effective Hilbert space.

[22] S. Tomsovic, Phys. Rev. Lett. 77, 4158 (1996).

[23] M. Wilkinson, Phys. Rev. A 41, 4645 (1990).

FIG. 1. The approximated spectrum (solid lines) obtained from eq. (2) is compared with the exact spectrum (dots).
FIG. 2. (a) The spectrum of the tranformed Hamiltonian by elimination of all avoided crossing satisfying eq. (四) with $v=1.5$. The scarred states corresponding to Fig. 3 (b) are shown. (b) Idem (a) for $v=5$.

FIG. 3. (a) Linear density plots of the eigenfunctions for the stadium billiar with $\ell=1$ and area $1+\pi / 4$. The numbers below each plot are the label (left) and the wave number $k$. (b) Linear density plots of the eigenfunctions for the transformed Hamiltonian by elimination of all avoided crossing satisfying eq.(4), with $v=5$. Letters on the top identify periodic orbits of Fig. 4 with higher contribution to each localized state. The label, $\sqrt{\left\langle k^{2}\right\rangle}$ and the dispersion $\sigma$ are showm below each plot. $\sigma$ is measured in units of the mean energy levels.

FIG. 4. Several short periodic orbits of the desymmetrized stadium billiard with $\ell=1$. 


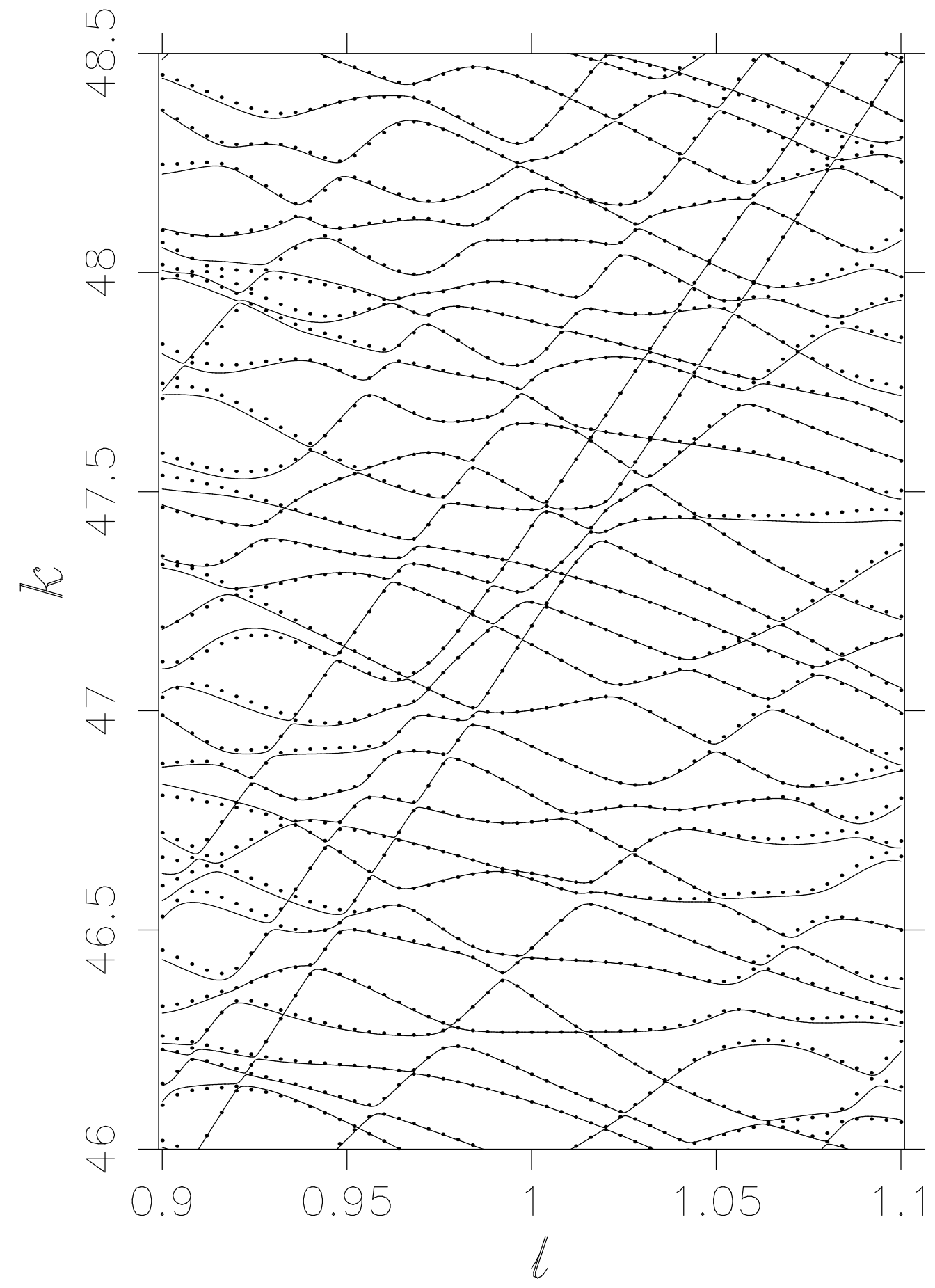


(a)

$\begin{array}{lll}23 & 18 & 14\end{array}$

26

28
12
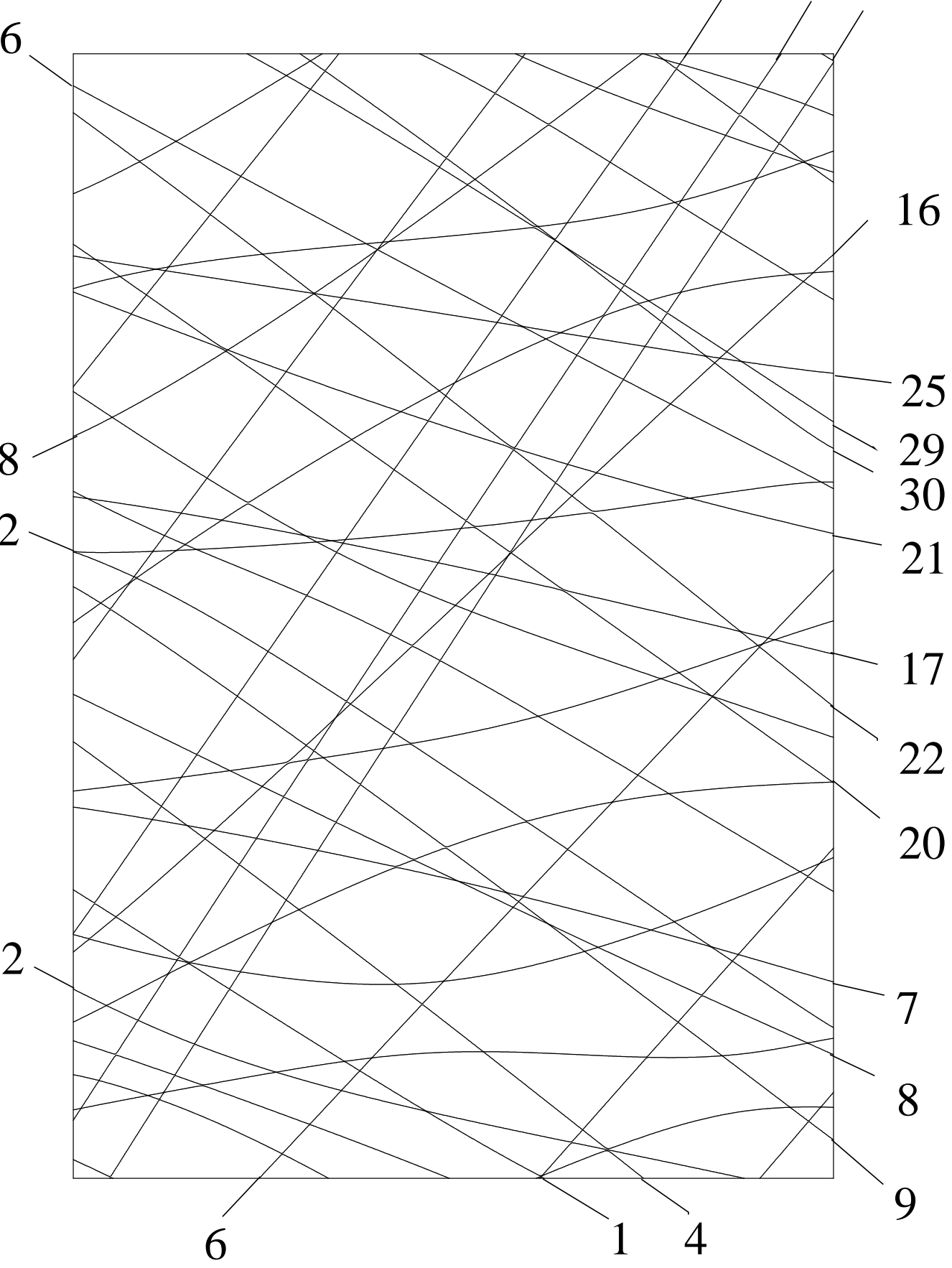
(b)

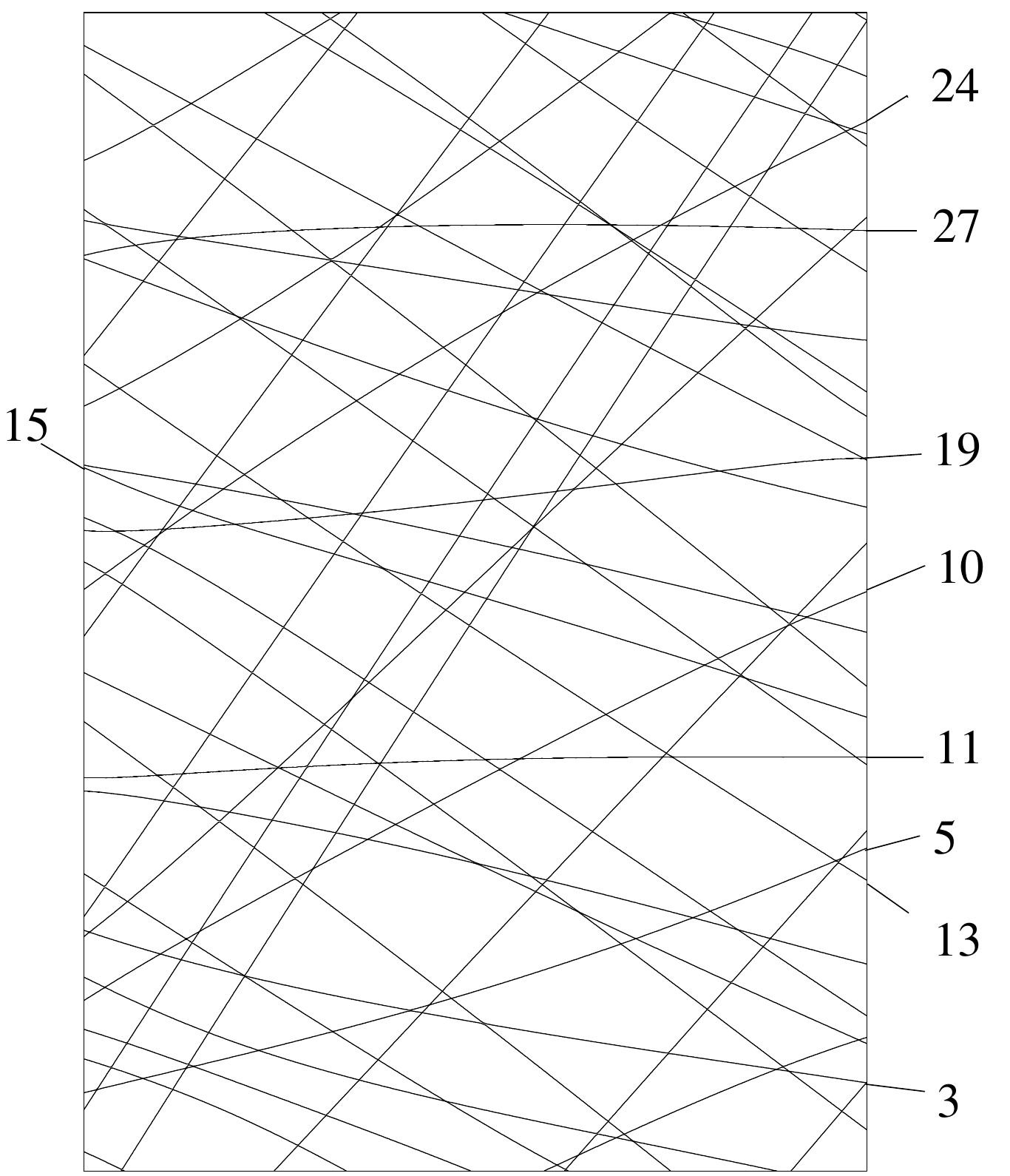


This figure "fig3a.gif" is available in "gif" format from: http://arXiv.org/ps/chao-dyn/9804011v1 
This figure "fig3b.gif" is available in "gif" format from: http://arXiv.org/ps/chao-dyn/9804011v1 

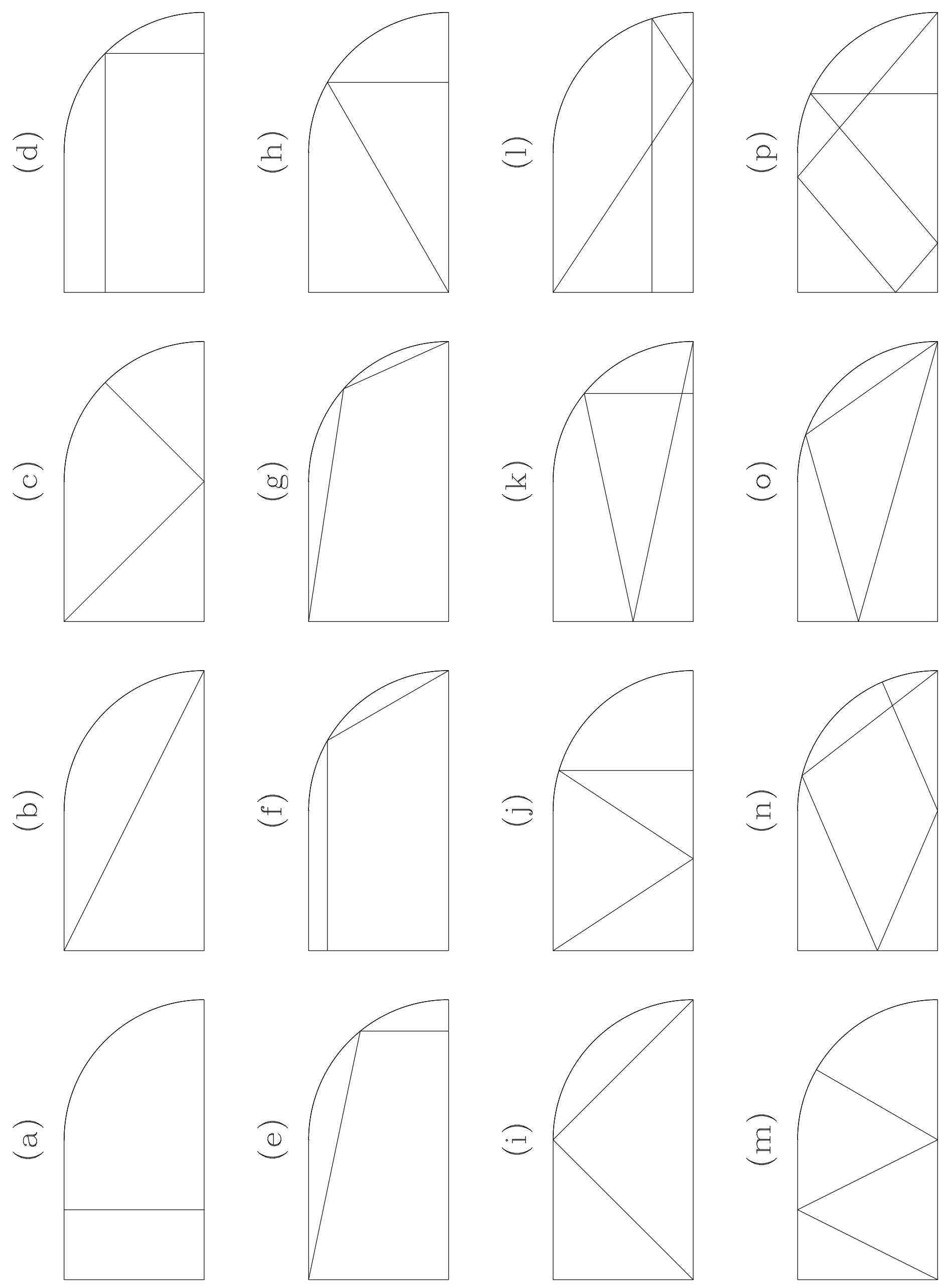